\title{
Short communication Endocrine and targeted treatments for breast cancer
} Clifford A Hudis

Memorial Sloan-Kettering Cancer Center, New York, New York 10065, USA

Corresponding author: Clifford A Hudis, hudisc@mskcc.org

Published: 20 December 2007

This article is online at http://breast-cancer-research.com/content/9/S2/S25 (c) 2007 BioMed Central Ltd

Endocrine therapy for breast cancer is arguably the first effective systemic treatment for human cancer and is the first targeted therapy [1]. Similar to many recently developed targeted therapies for breast and other malignancies, hormonal treatments for breast cancer include ligand deprivation (oestrogen depletion) and receptor agonism and antagonism. Given the safety and efficacy of the established hormonal options, the growing challenge that we confront with the development of an increasing number of novel targeted agents is whether these can be combined rationally, and if so then how, to improve patient outcomes while avoiding unwarranted toxicities.

Historically, the usual argument for combining therapeutics was the belief that two or more treatments would attack a malignant cell in an additive or synergistic manner, thereby improving outcomes [2]. Most combinations such as multiagent chemotherapy regimens (for instance, cyclophosphamide, methotrexate, and 5-fluouracil [CMF]) or chemotherapy with concurrent hormone therapy were empiric rather than truly translational and have not been shown to provide consistent advantage over sequential applications of optimal single agents and, in some cases, have been associated only with increased toxicities [3]. In breast cancer this paradigm was changed most clearly with the development of trastuzumab, which included translation of preclinical models into a rational human trial demonstrating improved survival compared with the sequential application of the same agents (chemotherapy and trastuzumab) [4,5]. With increased understanding of the roles not only of HER2 but also of the other members of the human epidermal growth factor receptor family (HER1, HER3, and HER4), rationale for specific combinations of hormonal therapies and signal transduction inhibitors (antibodies, tyrosine kinase inhibitors, and so on) has emerged [6]. However, a key challenge is to identify the most promising of these combinations for testing in a time of limited resources while simultaneously remaining sensitive to the risks to cost-effectiveness and quality of life that some combinations may represent. Because hormone therapy for breast cancer is generally well tolerated, any
Breast Cancer Research 2007, 9(Suppl 2):S25 (doi:10.1186/bcr1823)

negative impact on patient quality of life will have to be justified by meaningful improvements in outcome.

A first consideration could be the addition of agents that inhibit or reverse the development of hormone agent specific resistance. One model for such resistance suggests that bidirectional crosstalk between the oestrogen receptor and HER2 converts tamoxifen into an agonist [7]. This suggests that anti-HER2 therapies may be useful in preventing or reversing tamoxifen resistance specifically and hormone resistance in general. Based on such models, trastuzumab has been combined with modern hormone therapies in a variety of trials, including a large phase 3 study of the aromatase inhibitor anastrozole [8]. This randomized trial illustrates the challenges of such combinations, because although it demonstrated a statistically significant improvement in progression-free survival, this was not associated with improved overall survival and the clinical outcomes remained disappointing. Furthermore, the study left unresolved the potential value of sequential use of trastuzumab after progression on the aromatase inhibitor. Nevertheless, clinical trials are appropriately continuing in this vein, including, for example, fulvestrant with trastuzumab.

More recently, oral tyrosine kinase inhibitors, with varying degrees not only of anti-HER2 effectiveness but also of antiHER1 activity, have reached the clinic for breast cancer, led first by lapatinib [9]. The rationale for building combinations with hormonal agents is perhaps increased for these new drugs compared with trastuzumab because of their relative pan-HER inhibition. The Cancer and Leukaemia Group B has recently launched study 40302, a randomized double-blind placebo controlled trial of fulvestrant (a selective oestrogen receptor downregulator) alone or with lapatinib. Related trials including aromatase inhibition with or without gefitinib have not yet demonstrated clear clinical advantages, but they have provided tumour specimens that allow us to try to elucidate where and how such combinations might be effective $[10,11]$. Correlative science studies such as these are an important aspect of this generation of trials because they 
provide an opportunity to move our field beyond the pure empiric approach of earlier eras.

Among many enlightening examples of the value of this approach are the studies of cyclo-oxygenase (COX)-2 inhibitors. Preclinical studies suggested a role for these agents in a variety of tumour settings, including hormoneresponsive breast cancer. Based on this, a factorial design was incorporated into the National Cancer Institute of Cancada ( $\mathrm{NCIC}) \mathrm{MA}-27$ trial comparing anastrozole with exemestane, such that patients were also randomized for the use of celecoxib. After the potential toxicities of celecoxib in the general population were reported, this randomization was dropped. However, COX inhibition, both COX-1 and COX-2, remains an important area for clinical research in breast cancer for the following reasons. First, COX inhibitors (aspirin and nonsteroidal anti-inflammatory agents) are widely used in the general population. Second, COX-2 expression is associated with HER2 overexpression. Finally, there is a connection between COX-2 and aromatase activity [12,13]. To better understand this latter connection, a preoperative or so-called 'window' trial allows for a brief exposure to celecoxib with paired specimen collection to determine whether the target is in fact inhibited in breast cancer in vivo, accompanied by the predicted downstream effects. Such trials will gain importance as we attempt to identify the best of the increasing number of new targeted agents for clinical testing. Validation of targeting in vivo should be a component of new drug development whenever possible, but will require dedicated collaborative efforts supported by adequate resources.

Angiogenesis has long been recognized as a critical step in the development of human cancer and inhibitors, both antibody based (bevacizumab) and in the form of oral tyrosine kinase inhibitors (for instance, sunitinib, sorafenib and others), are now broadly available for clinical testing [14]. Animal models demonstrating the role of oestrogens in stimulating angiogenesis provide the foundation of testing combinations of anti-angiogenics with hormone therapy in breast cancer [15-17]. Because the first narrowly targeted anti-angiogenic monoclonal antibody, bevacizumab, has demonstrated broad antitumour activity accompanied by a novel toxicity profile, a phase 2 study of this drug combined with an aromatase inhibitor was conducted to establish safety in preparation for a phase 3 trial [18]. The latter study is now set to launch in metastatic breast cancer, as are several similar studies utilizing vascular endothelial growth factor receptor tyrosine kinase inhibitors. If the toxicity of these combinations is acceptable in large numbers of patients, then such combinations could represent an important improvement over conventional single agent hormonal approaches.

Clearly, we are at the beginning of a new era of hormone therapy trials. Instead of continuing only to develop novel hormone therapies or 'better' versions of existing agents, we are instead asking biological questions such as how anti-HER agents with demonstrated effectiveness combined in preclinical models with hormonal interventions will fare in the clinic. We are asking as well about the potential efficacy of inhibitors of downstream signalling mediators (for example, mammalian target of rapamycin [mTOR] inhibition) and entirely novel targeted therapies focused not on the tumour per se but on the stroma and supporting tissues (anti-angiogenic agents). These studies should provide more biological information than the empirical studies of yesteryear and we should view the results critically, making certain that whatever quantitative benefits we observe are clinically justified.

\section{Acknowledgement}

This article has been published as part of Breast Cancer Research Volume 9 Supplement 2, 2007: Controversies in Breast Cancer. The full contents of the supplement are available online at http://breastcancer-research.com/supplements/9/S2.

\section{References}

1. Beatson GT: On the treatment of inoperable carcinoma of the mamma: suggestions for a new method of treatment, with illustrative cases. Lancet 1896, ii:104-107.

2. Cannellos GP, DeVita VT, Gold GL, Chabner BA, Schein PS, Young RC: Cyclical combination chemotherapy for advanced breast cancer. BMJ 1974, 1:218-220.

3. Sledge GW, Neuberg D, Bernardo P, Ingle JN, Martino S, Rowinsky EK, Wood WC: Phase III Trial of doxorubicin, paclitaxel, and the combination of doxorubicin and paclitaxel as frontline chemotherapy for metastatic breast cancer: an Intergroup Trial (E1193). J Clin Oncol 2003, 21:588-592.

4. Slamon DJ, Leyland-Jones B, Shak S, Fuchs H, Paton V, Bajamonde A, Fleming T, Eiermann W, Wolter J, Pegram M, et al.: Use of chemotherapy plus a monoclonal antibody against HER2 for metastatic breast cancer that overexpresses HER2. $N$ Engl J Med 2001, 344:783-792.

5. Hudis C: Drug therapy: trastuzumab. N Engl J Med 2007, 357: 39-51.

6. Dowsett M, Harper-Wynne C, Boeddinghaus I, Salter J, Hills M, Dixon M, Ebbs S, Gui G, Sacks N, Smith I: HER-2 amplification impedes the antiproliferative effects of hormone therapy in estrogen receptor-positive primary breast cancer. Cancer Res 2001, 61:8452-8458.

7. Shou J, Massarweh S, Osborne CK, Wakeling AE, Ali S, Weiss H, Schiff R: Mechanisms of tamoxifen resistance: increased estrogen receptor-HER2/neu cross-talk in ER/HER2-positive breast cancer. J Natl Cancer Inst 2004, 96:926-935.

8. Kaufman B, Mackey J, Clemens M, Bapsy P, Vaid A, Wardley A, Tjulandin S, Jahn M, Lehle M, Jones A: Trastuzumab plus anastrozole prolongs progression-free survival in postmenopausal women with HER2-positive, hormone-dependent metastatic breast cancer (MBC). In Proceedings from the 31 st ESMO Congress, Istanbul 2006. Abstract LBA2.

9. Geyer CE, Forster J, Lindquist D, Chan S, Romieu CG, Pienkowski T, Jagiello-Gruszfeld A, Crown J, Chan A, Kaufman B, et al:: Lapatinib plus capecitabine for HER2-positive advanced breast cancer. N Engl J Med 2006, 355:2733-2743.

10. Kurebayashi J, Okubo S, Yamamoto Y, Sonoo H: Inhibition of HER1 signaling pathway enhances antitumor effect of endocrine therapy in breast cancer. Breast Cancer 2004, 11:38-41.

11. Johnston SR, Head J, Pancholi S, Detre S, Martin LA, Smith IE, Dowsett $M$ : Integration of signal transduction inhibitors with endocrine therapy: an approach to overcoming hormone resistance in breast cancer. Clin Cancer Res 2003, 9:524S-532S.

12. Subbaramaiah K, Howe LR, Port ER, Brogi E, Liu CH, Hla T, Dannenberg AJ: Cox-2-derived prostaglandins regulate aromatase activity in breast cancer [abstract 1940]. In Proceedings of the American Association for Cancer Research; 11-14 July 2003; Washington, DC. Philadelphia, PA: American Association for Cancer Research; 2003:44. 
13. Subbaramaiah $\mathrm{K}$, Howe LR, Port ER, Brogi E, Fishman J, Liu CH, Hla T, Hudis C, Dannenberg AJ: HER-2/neu status is a determinant of mammary aromatase activity in vivo: evidence for a cyclooxygenase-2-dependent mechanism. Cancer Res 2006, 66:5504-5511.

14. Hanahan D, Folkman J: Patterns and emerging mechanisms of the angiogenic switch during tumorigenesis. Cell 1996, 86: 353-364.

15. Shweiki D, Itin A, Neufeld G, Gitay-Goren H, Keshet E: Patterns of expression of vascular endothelial growth factor (VEGF) and VEGF receptors in mice suggest a role in hormonally regulated angiogenesis. J Clin Invest 1993, 91:2235-2243.

16. Albrecht ED, Aberdeen GW, Niklaus AL, Babischkin JS, Suresch DL, Pepe GJ: Acute temporal regulation of vascular endothe- lial growth/permeability factor expression and endothelial morphology in the baboon endometrium by ovarian steroids. $J$ Clin Endocrinol Metab 2003, 88:2844-2852.

17. Jain RK, Safabakhsh N, Sckell A, Chen Y, Jiang P, Benjamin L, Yuan $F$, Keshet $E$ : Endothelial cell death, angiogenesis, and microvascular function after castration in an androgen-dependent tumor: role of vascular endothelial growth factor. Proc Natl Acad Sci USA 1998, 95:10820-10825.

18. Traina TA, Dickler MN, Caravelli JF, Yeh BM, Brogi E, Panageas $K$ Flores SA, Norton L, Hudis C, Rugo H: A phase II trial of letrozole in combination with bevacizumab, an anti-VEGF antibody, in patients with hormone receptor-positive metastatic breast cancer [abstract]. Breast Cancer Res Treat 2005, 94(Suppl 1): A2030. 